\title{
Fundamentals towards Adaptive Fractal Filtering for Multiple Dimensional Signals
}

\author{
Zhuang Mo and Liang Gong* \\ School of Mechanical Engineering, Shanghai Jiao Tong University, China \\ mozh-mcfly@foxmail.com,gongliang_mi@sjtu.edu.cn \\ *The corresponding author
}

Keywords: Adaptive filter; Fractal dimension; Fuzzy control; Filter square error

\begin{abstract}
The fractal dimension of signal can be applied into signal filtering. This application makes filtering adaptive to the change of the signal condition. To compare different filtering effect with different relation between filter parameter and signal fractal dimension, this paper shows results of two experiments, which include the processing of one-dimensional signal and image (two-dimensional signal). In these experiments, the outputs of fractal filter with different parameters are compared, showing filter parameters determined by signal fractal dimension in different ways are suitable in different ranges respectively in Weierstrass signal process, and that different relation between filter parameter and image fractal dimension can bring different edge enhancement effect.
\end{abstract}

\section{Introduction}

Yan Hao, Taiyong Wang, Jian Wan and i.e. [1] analyzed the relation between the fractal box dimension and signal-noise ratio of certain kinds of signals, showing that they have linear relation when SNR is in certain range. Liang Chen, Xiongwei Zhang [2], Yaqiang Shen, Genliang Feng [3] applied the fractal dimension on time domain into speech signal segment. Yue Liu, Xiaoting Wang [4] applied the fractal dimension on frequency domain into speech end point detection. Yuyi Cheng, Jie Yu and Jianyi Zhong [5] applied the fractal box dimension into adjusting the window width of the median filter.

And the fractal dimension can also be applied together with fuzzy control filter. Yueliang Chen and Hongyang Guo [6] applied the fractal filter into Weierstrass signal process, during which the signal is divided into frames. In BU Heerdun and Feng Han's [7] study, they process the signal as an integrate part. Furthermore, they also offered an algorithm related to fractal dimension of images, which they demonstrated to be effective in enhance the contour in images [7]. Minjin Wu [8] applied fractal filtering into speech signal filtering, and used the filtering square error as criterion to check the effect of the fractal filter. Zhongyi Deng [9] also applied the fractal filter into speech signal process, with different relation between the parameter of the filter and the fractal dimension of the signal.

Their studies show the advantage of fractal filtering over non self-adaptive filter. The experiments in this paper aims to make more specific comparisons, and show the difference effect caused by different parameter determination.

\section{Fractal Dimension}

The definition of fractal grid dimension can be described with the equations below [3][6-10]:

$$
\begin{aligned}
& D^{(k)}(\Delta)=\sum_{j=1}^{n}\left|x_{j}-x_{j+1}\right| \\
& D^{(k)}(2 \Delta)=\sum_{j=1}^{n / 2}\left(\max \left\{x_{2 j-1}, x_{2 j}, x_{2 j+1}\right\}-\min \left\{x_{2 j-1}, x_{2 j}, x_{2 j+1}\right\}\right)
\end{aligned}
$$




$$
\begin{aligned}
& N^{(k)}(\Delta)=D^{(k)}(\Delta) / \Delta \\
& N^{(k)}(2 \Delta)=D^{(k)}(2 \Delta) / 2 \Delta \\
& d_{F}^{(k)}=\frac{\log N^{(k)}(\Delta)-\log N^{(k)}(2 \Delta)}{\log 2}=1+\log _{2} \frac{D^{(k)}(\Delta)}{D^{(k)}(2 \Delta)}
\end{aligned}
$$

Here, $k$ means the sequence number of the frame in the entire signal. $N$ means the number of discrete points in each frame. $\Delta$ is the side length of the grid used to calculate the fractal dimension.

\section{Fuzzy Control Filter}

In the relative studies, a fuzzy control digit filter is introduced [2-3][6-10]:

$$
y_{i}=a x_{i}+(1-a) y_{i-1}
$$

The $a$ in Eq. 6 is the fuzzy control parameter, which can connect fractal dimension with fuzzy filter through the equations [6-8][10]:

$$
\begin{aligned}
& a=(2-d)^{2} \\
& a=2-2^{d-1} \\
& a=d \cdot(2-d) \\
& a=2-d
\end{aligned}
$$

\section{Fractal Filtering Experiments}

Weierstrass Signal Processing. Yueliang Chen and Hongyang Guo [6] used Weierstrass function, of which the high frequency components can be regarded as noise, as an input signal to the filter to generate output signal as a result of fractal filtration.

The expression of Weierstrass function [6]:

$$
f(t)=\sum_{k=1}^{N} \lambda^{(s-2) k} \sin \left(\lambda^{k} t\right)
$$

According to Chen's experiment [6], $\lambda=1.5, s=1.5, N=100$. Then, a signal from initial time (0) to $6.5536 \mathrm{~s}$, with sampling interval of $0.0064 \mathrm{~s}$ is generated as an input signal in the experiment. This signal will be divided into several frames that consists of 128 data points. The fuzzy control number will be determined by the fractal dimension of the frame, however, the quantitative relation is not specified. So we use every Eq.7 to Eq.10 to filter the signal, and compare the results with that of the cases in which $a=0.7$ and 0.15 .

The filtering square error, the square error between the original signal and the output of the filter, can be considered as the criteria of the filtering effect [8][10]. The effect of the filter is better when the error is smaller.

We can set the value of $N$ between 1 and 99. The sinusoidal components of Weierstrass signal with higher order will be regard as noise and the rest of the components form the original signal. Different values of $N$ cause different signal-noise ratio. Hence, we can generate a sequence of square error corresponding to different values of $N$ (Fig. 1): 


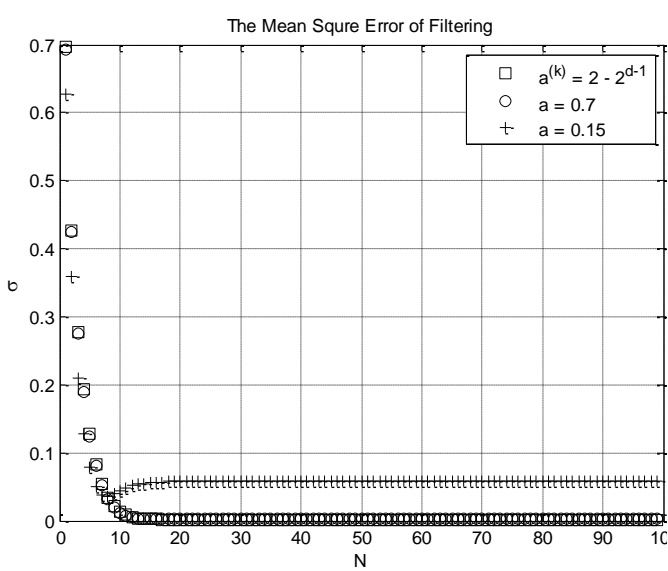

Figure 1. Mean square error with $N$ from 1 to 99

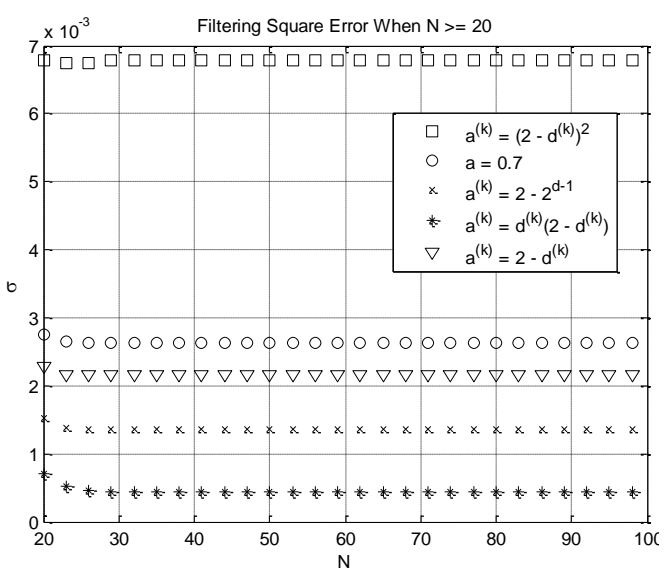

Figure 2. Mean square error with $N$ from 20 to 98 (with step length of 3)

For Eq.7, Eq.9 and Eq.10, we can get similar image indicating that the constant control parameter 0.15 only leads to better effect when noise is loud. However, when noise becomes small, the constant control parameter 0.15 causes obvious larger error, for a constant parameter is not able to adjust to the change of the signal. The control parameter of constant value 0.7 and the control parameter determined by fractal dimension give similar results in most cases when $N>20$, where the noise is small. Then, we compare the sequence generated according to Eq. 7 to Eq.10 from $N=20$, and the sequence generated by constant control parameter 0.7 (Fig. 2).

Fig. 2 shows that different relation between control parameter and the short-time fractal dimension causes different effect of the filtering. In this case of Weierstrass function, Eq.9 gives best value of the control parameter when noise is small.

Then, we compare them with $\mathrm{N}$ in the range from 1 to 20 :

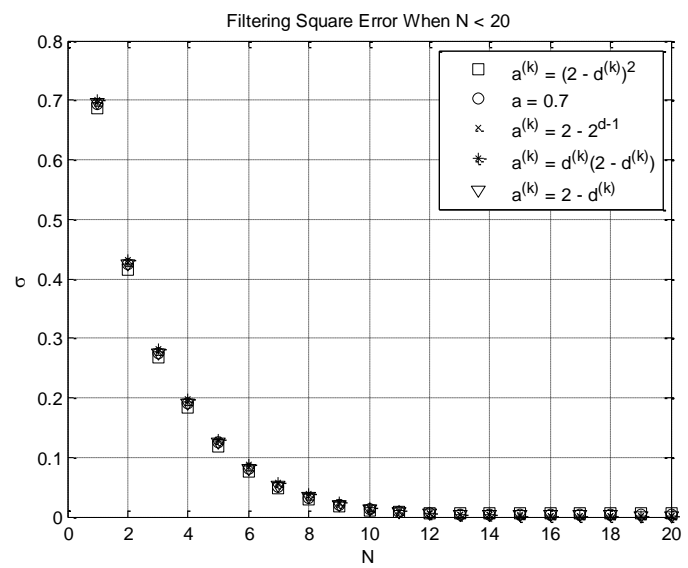

Figure 3. Mean square error with $N$ from 1 to 20

The control parameter determined by Eq.7 loses its advantage when $N$ is larger than 10, before which the parameter determined by Eq.7 gives best effect of the filtering. Hence, the specific relation between the fuzzy control parameter and the fractal dimension of the signal suitable to be applied in filtering depends on the concrete conditions of the signal.

Image Processing. BU Heerdun and Feng Han also provided method of image processing, including the relation of the filter parameter and the fractal dimension of the image [7]:

$a=(3-d)^{6}$

And the value of each pixel [7][8]: 


$$
g\left(x_{i}, y_{i}\right)=\alpha f\left(x_{i}, y_{i}\right)+\frac{1-\alpha}{3}\left[g\left(x_{i-1}, y_{i-1}\right)+g\left(x_{i}, y_{i-1}\right)+g\left(x_{i-1}, y_{i}\right)\right]
$$

Then, take 10 rows and 10 columns of pixels as 20 lines of one-dimensional data. Calculate their average fractal dimension, which can be regard as the fractal dimension of the whole image [7]. Compare the original picture and the processed picture, it's obvious that the contours or edges are enhanced in the processed image.

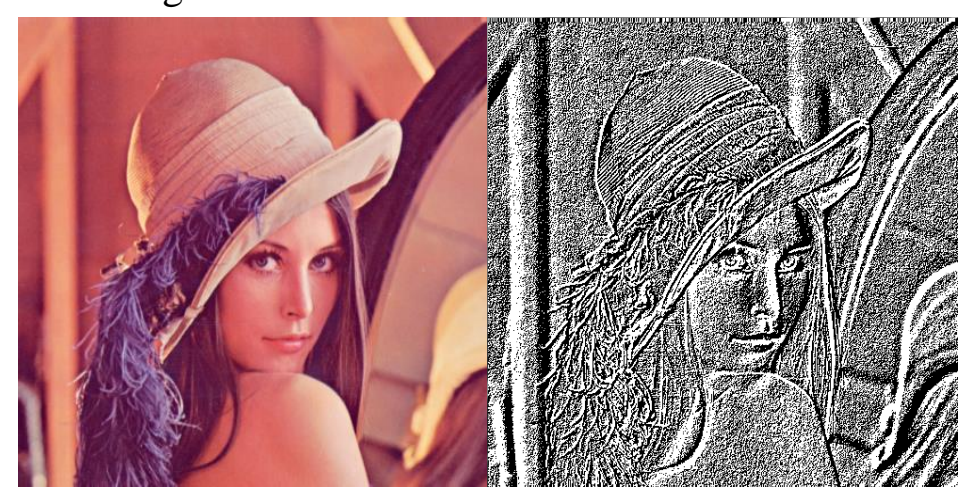

Figure 4. Image processing origin image (left) from Internet: https://en.wikipedia.org/wiki/Lenna\#/media/File:Lenna.png; Processed image (right)

Make adjustment on Eq.12:

$a=(3-d)^{p}$

Process the same image with different index number $p$ :

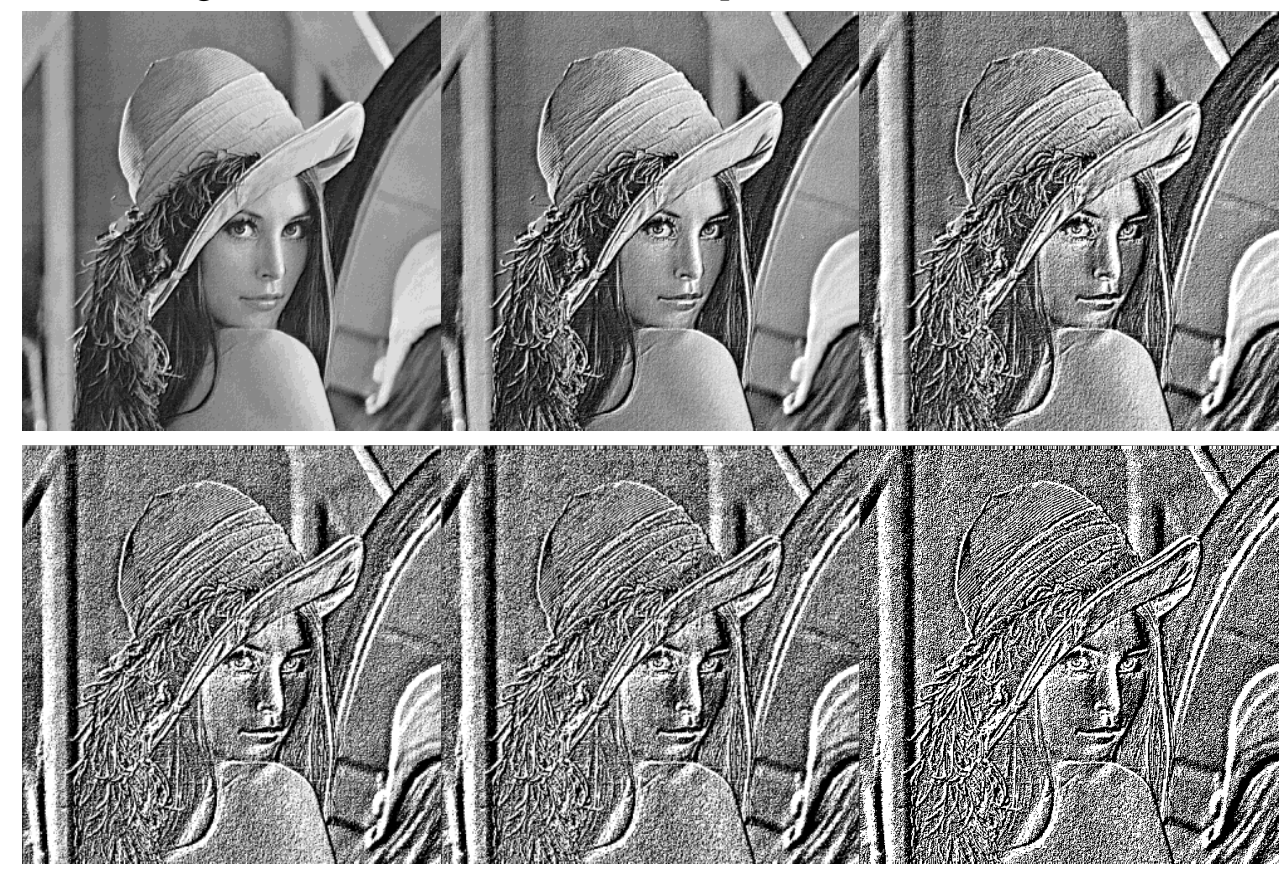

Figure 5. Output images with $p$ from 1 to 6 (from left to right)

When the value of the index number is from 1 to 4 , it is obvious that the effect of edge enhancement is increasingly distinct with a growing index number.

\section{Summary}

The results of the experiments show that a properly estimated fuzzy control parameter can connect fractal dimension and signal filtering very well. The fractal dimension will make the filter adaptive 
to the change of input signal. However, the effect of a fractal filter depends on the condition of the signal to be processed, not only the filter itself. In the processing of Weierstrass signal, different relations between fuzzy control parameter and the short-time fractal dimension of the signal have advantage respectively in different ranges. And about image processing, the experiment shows that different index number can bring different effect of the edge enhancement.

\section{Acknowledgement}

This work was partially supported by the National Scientific Foundation of China under Grant NO. 11202125 .

\section{References}

[1] Y. Hao, T.Y. Wang, J. Wan, P. Zhang and L. Liu: Chinese Journal of Scientific Instrument, Vol.32 (2011) No.3, p.540-545 (In Chinese)

[2] L. Chen and X.W. Zhang: Journal of Beijing University of Posts and Telecommunications, Vol.26 (2003) Sup, p.112-125 (In Chinese)

[3] Y.Q. Shen and G.L. Feng: Journal of Zhejiang Normal University (Nat.Sci), Vol.22 (1999) No.1, p.16-21

[4] Y. Liu and X.T. Wang: Microelectronics \& Computer, Vol.32 (2015) No.9, 81-89 (In Chinese)

[5] Y.Y. Cheng, J. Yu and J.Y. Zhong: Chinese Journal of Analytical Chemistry, Vol.29 (2001) No.11, p.1246-1250 (In Chinese)

[6] Y.L. Chen and H.Y. Guo: Journal of North University of China (Natural Science Edition), Vol.27 (2006) No.3, p.272-275 (In Chinese)

[7] H. Bu and F. Han: Progress in Geophysics, Vol.23 (2008) No.2, p.627-630 (In Chinese)

[8] M.J. Wu: Introduction to Fractal Information (Shanghai science and Technology Literature Publishing House, China 1994), p.182-189 (In Chinese)

[9] Z.Y. Deng: Chinese Journal of Electron Devices, Vol.29 (2006) No.2, p.391-400 (In Chinese)

[10] W. Ouyang, Y. He and W. Fang: Journal of System Simulation, Vol.18 (2006) No.3, p.680-684 (In Chinese) 